\title{
Orbits for five southern visual binaries
}

\author{
R. W. Argyle ${ }^{1}$, A. Alzner ${ }^{2}$, and E. P. Horch ${ }^{3}$ \\ 1 Institute of Astronomy, Madingley Road, Cambridge CB3 0HA, UK \\ 2 Zeckerner Hauptstrasse 3, 91334 Hemhofen, Germany \\ e-mail: Aalzner@aol.com \\ 3 Chester F. Carlson Center for Imaging Science, Rochester Institute of Technology, 54 Lomb Memorial Drive, \\ Rochester, NY 14623-5604, USA \\ e-mail: horch@cis.rit.edu
}

Received 16 July 2001 / Accepted 18 December 2001

\begin{abstract}
First orbits have been calculated for the visual binaries WDS 00090-5400, 01061-4643, 05248-5219 and 08447-5443 (Alzner \& Argyle 2000; Argyle \& Alzner 2000). The current period for the orbit of WDS 18068-4325 (Wierzbinski 1958) is too short to represent recent observations. The period has been lengthened to 450 years (Alzner \& Argyle 2001). For 00090-5400 the period is 221 years with a moderate eccentricity; 01061-4643 is currently near periastron but is underobserved; 05248-5219 has passed periastron but there have been no observations since 1993. The visual component of WDS 08447-5443 (= $\delta$ Vel), which is not the same as the eclipsing component recently discovered by Otero (2000), has an unexpectedly short period.
\end{abstract}

Key words. astrometry - binaries: close - binaries: visual - techniques: interferometric

\section{Introduction}

Measurement of visual double stars in the southern hemisphere, whether it be by the classical micrometer method, or the use of speckle interferometry, has been at a low ebb during the past 20 years or more. That a 2 nd magnitude star, $\delta$ Vel, known to be a binary for more than 100 years has recently passed virtually unobserved through periastron is indicative of this fact. In addition, the Hipparcos satellite has discovered more than 1000 new systems south of -30 degrees all of which will need to be followed up at some stage.

In an attempt to redress the balance one of us, $\mathrm{EH}$, (see, for instance, Horch et al. 1996) has been carrying out a programme of speckle interferometry designed to monitor the urgent systems in the southern sky. A search through the WDS catalogue (Mason et al. 2001) revealed a number of visual binaries which have shown significant motion and which need to be kept under regular observation.

The derivation of stellar masses still relies almost entirely on the observation of binary star orbits. Four out of the five systems under discussion have reached critical points in their orbits and need to be brought to the attention of observers. Discussion of the orbital motion to

Send offprint requests to: R. W. Argyle,

e-mail: rwa@ast.cam.ac.uk date in each case yields reasonable preliminary orbital solutions, allowing some investigation of the system masses and the spectral types of the individual components.

\section{Method}

For the weights $\mathrm{w}$ assigned to the observations the rule $w=1 / \sigma^{2}$ was applied where $\sigma$ is the error of observation. Errors for the visual observations were assumed to follow the rule $C \rho^{\frac{1}{3}}$, where $C$ for a single measurement is $0 . .03$ in position angle $(\rho \mathrm{d} \theta)$ and $0 . .08$ in separation $(\mathrm{d} \rho)$ (Heintz 1978). Some early visual measurements received much lower weights because of obviously large deviations. The linear measurement uncertainty for a single speckle observation generally varies between 0.005 and $0 . \prime 01$.

First initial elements have been derived for each system using a graphical method. Least squares fits were then applied using the technique of differential correction in polar coordinates. In each case the least squares fit converged and it was possible to correct all seven Campbell elements simultaneously. If handled with care and applying reasonable corrections in each step, the differential correction can change the initial elements considerably.

Afterwards errors for the elements were calculated from the covariance matrix and the residuals to all observations. In a third step for each pair the period $P$ was varied over large ranges and optimum orbits were calculated 
minimizing the sum of least squares with the individual periods being kept fixed. This procedure was performed for three reasons, checking for other possible minima of the objective function, checking the reliability of the errors calculated from the covariance matrix, and calculating upper and lower limits for the important quantity $a^{3} / P^{2}$ in order to calculate mass errors.

As a result the orbital elements stayed unchanged, but for some cases the values for the errors needed large revisions. For example, for I 345 the covariance matrix plus residuals gave an error of +127 and -54 years, but reliable fits could be obtained even for periods up to 450 years leading to an uncertainty of +259 years for the upper limit. In the case of HJ 5014 periods shorter than 353 years can fit the observations after 1880 reasonably well, but the calculated distances for the important 1836 and 1857 observations become too short.

The mass errors $\mathrm{d} M$ were calculated from the errors $\mathrm{d} x$ for $x=a^{3} / P^{2}$ and the errors $\mathrm{d} \pi$ for the Hipparcos parallaxes $\pi$ :

$$
\begin{aligned}
\mathrm{d} M & =\sqrt{\left(\frac{\partial M}{\partial x} \cdot \mathrm{d} x\right)^{2}+\left(\frac{\partial M}{\partial \pi} \cdot \mathrm{d} \pi\right)^{2}} \\
& =\sqrt{\left(\frac{1}{\pi^{3}} \cdot \mathrm{d} x\right)^{2}+\left(\frac{-3 a^{3}}{\pi^{4} P^{2}} \cdot \mathrm{d} \pi\right)^{2}}
\end{aligned}
$$

The results of this error analysis are given in Table 3.

\section{Results and discussion}

In the discussion for each system which follows below we have employed the Baize-Romani method as formulated by Heintz (1978) to evaluate the dynamical parallax of each system. Heintz bases his formula on the slope of the Mass-Luminosity Relation (MLR) given by Cester (1965) and his own data. This slope is 3.8 for masses $>0.5 M_{\odot}$ whilst a recent investigation by Lampens et al. (1997) find the slope of the MLR to be 3.9 for $0.8 M_{\odot}>M>7.0 M_{\odot}$. As we are dealing with stars on the upper part of the MLR we have used this value in the calculation of the dynamical parallax using Baize-Romani.

In Table 1 we present the available astrophysical data for each pair and tabulate the mass for each system based on the Hipparcos parallax and on the derived dynamical parallax along with the mass errors based on the uncertainties in the orbital periods, semi-major axes and Hipparcos parallaxes. The $V_{\mathrm{J}}$ apparent magnitudes derived from the $(V-I)$ index, $H_{\mathrm{P}}$ and $\Delta H_{\mathrm{P}}(\mathrm{ESA}, 1997)$ are also given and absolute magnitudes are based on the Hipparcos $V_{\mathrm{J}}$ apparent magnitude, the Hipparcos parallax and a correction for absorption (van Herk 1965).

The results of the orbital analysis are given in Table 2, whilst the estimated errors in the derived orbital elements are listed in Table 3.

Table 4 gives an ephemeris for each system for the period 2001 to 2010. Table 5 contains the observations used in the calculation of the orbits, along with the residuals from the derived elements. All measurements have

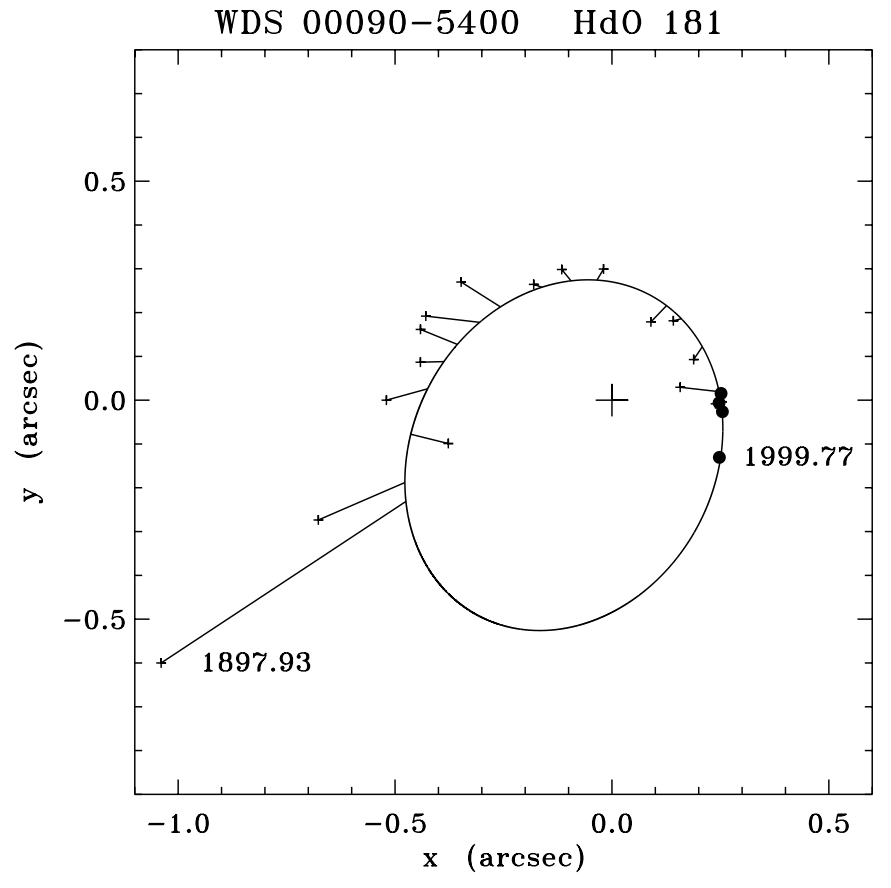

Fig. 1. The apparent orbit of HdO181, period $=221$ years. North is down, east is to the right. The epoch of observation is shown next to the first and last data points. (The same conventions are used in Figs. 2-5.)

been corrected for precession except for HdO181 where the correction is negligible. For those observations recorded as "single" the numbers in brackets represent the predicted PA and separation from the orbit for that epoch.

\section{WDS 00090-5400, HdO181, HIP 730}

This pair was first noted by W. B. Clymer (Pickering 1902) on 1897, Dec. 5 during a systematic search for double stars carried out with the 13-inch Boyden refractor at Arequipa in Peru. The distance slowly decreased and reached a minimum value of about 0 '. 20 in the early 1980 's. There are relatively few observations of this pair and it is largely thanks to the efforts of Dr. W. H. van den Bos that the current analysis is possible.

Using the Hipparcos parallax $(7 \pm 0.74$ mas $)$ and the orbital elements $a$ and $P$ given in Table 1 the mass sum for this pair is $4.4 M_{\odot}$. The WDS gives the spectral type as G4IV and the Bright Star Catalogue (Hoffleit 1983) indicates that the star is also a spectroscopic binary but gives no reference. The dynamical parallax is 7.0 mas, in excellent agreement with the trigonometric parallax found by Hipparcos.

Houk \& Cowley (1975) gives a spectral type of $\mathrm{G} 8 \mathrm{III}+\mathrm{A} / \mathrm{F}$ with the comment "composite-like spectrum" so it may be that this is the source to which the Bright Star Catalogue refers. Only one velocity has been found in the literature $\left(+0.9 \pm 0.2 \mathrm{~km} \mathrm{~s}^{-1}\right)$, (Evans et al. 1961). The Hipparcos $B-V$ is bluer than that expected for a G8 giant and along with a mass sum of about $4.6 M_{\odot}$ then it is reasonable to conclude that the companion is probably a late A main sequence star. 
Table 1. Astrophysical data.

\begin{tabular}{llllllllllll}
\hline Pair & $V(\mathrm{~A})$ & $V(\mathrm{~B})$ & $M_{v}(\mathrm{~A})$ & $M_{v}(\mathrm{~B})$ & $\begin{array}{l}\pi_{\text {Hipp }} \\
(\mathrm{mas})\end{array}$ & $\begin{array}{l}\epsilon_{\text {Hipp }} \\
(\mathrm{mas})\end{array}$ & $\Sigma M$ & Error(M) & $\begin{array}{c}\pi_{\text {dyn }} \\
(\mathrm{mas})\end{array}$ & $\Sigma M$ & Spectra \\
\hline HdO181 & 6.59 & 7.91 & 0.70 & 2.02 & 7.00 & 0.74 & 4.42 & $+2.0,-1.45$ & 7.0 & 4.8 & G4IV, (G8III) \\
Slr1 & 3.93 & 4.20 & -0.13 & 0.14 & 17.63 & 2.09 & 5.72 & $+2.50,-2.04$ & 17.2 & 6.7 & G8III \\
I 345 & 7.00 & 7.64 & 0.51 & 1.15 & 6.75 & 0.55 & 3.24 & $+2.93,-1.00$ & 5.4 & 5.6 & A0V, (A2V) \\
I 10 & 1.97 & 5.55 & 0.02 & 3.60 & 40.90 & 0.38 & 5.71 & $+1.27,-1.08$ & 44.3 & 4.7 & A1V \\
HJ 5014 & 5.63 & 5.71 & 2.40 & 2.48 & 22.79 & 1.12 & 3.54 & $+0.94,-0.80$ & 23.0 & 3.7 & A5V, A5V \\
\hline
\end{tabular}

Table 2. Orbital elements for five visual binaries.

\begin{tabular}{llllll}
\hline Elements & HdO181 & Slr1 & I 345 & I 10 & HJ 5014 \\
\hline$P($ yr $)$ & 221 & 195 & 191 & 142 & 450 \\
$T$ & 1978.71 & 2003.9 & 1994.17 & 2000.80 & 1854.7 \\
$e$ & 0.406 & 0.66 & 0.64 & 0.47 & 0.65 \\
$a(\operatorname{arcsec})$ & 0.42 & 1.06 & 0.30 & 1.99 & 2.04 \\
$i(\mathrm{deg})$ & 154.1 & 134.3 & 142.7 & 105.2 & 123.1 \\
$\omega(\mathrm{deg})$ & 25.3 & 294.3 & 38.4 & 188.0 & 282.7 \\
$\Omega_{2000}(\mathrm{deg})$ & 161.6 & 137.8 & 52.1 & 163.6 & 85.8 \\
\hline
\end{tabular}

Table 3. Errors of orbital elements obtained from covariance matrix and residuals, and calculation of alternative orbits by varying the period $P$.

\begin{tabular}{llllll}
\hline Elements & HdO181 & Slr1 & I 345 & I 10 & HJ 5014 \\
\hline $\mathrm{d} P(\mathrm{yr})$ & $+116,-40$ & $+119,-35$ & $+259,-54$ & $+14,-11$ & $+170,-97$ \\
$\mathrm{~d} T(\mathrm{yr})$ & 1.9 & 1.3 & 2.3 & 1.6 & 2.9 \\
$\mathrm{~d} e$ & 0.12 & 0.05 & 0.05 & 0.02 & 0.06 \\
$\mathrm{~d} a(\operatorname{arcsec})$ & $+0.20,-0.06$ & $+0.51,-0.14$ & $+0.20,-0.01$ & $+0.03,-0.01$ & $0.66,-0.41$ \\
$\mathrm{~d} i(\mathrm{deg})$ & 14 & 15 & 10 & 2.2 & 6 \\
$\mathrm{~d} \omega(\mathrm{deg})$ & 11 & 15 & 10 & 14 & 10 \\
$\mathrm{~d} \Omega(\mathrm{deg})$ & 9 & 8 & 28 & 1.3 & 0.9 \\
\hline
\end{tabular}

\section{WDS 01061-4643, Slr 1, HIP 5165}

The bright star $\beta$ Phe was found to be double by Sellors (1893) using the 11.5-inch refractor at Sydney Observatory in 1891.

Although no double star astrometry or magnitude difference appears in the main Hipparcos catalogue, a general note at the end of vol. 5 (page GN3) gives a relative position for 1991.25 which was not used in the orbital analysis but which gives small residuals (see Table 4) and $H_{\mathrm{P}}=0.27\left(V_{\mathrm{J}}=0.27\right)$. Whilst the Hipparcos parallax given in the catalogue is subject to a large uncertainty $(16.46 \pm 22.22$ mas $)$, the note above also gives a "more likely solution" of $17.63 \pm 2.09$ mas and this value has been adopted here.

Houk (1978) gives a spectral type of G8III whilst Simbad lists $B=4.199$ and $V=3.323$ giving $B-V=$ 0.876 a little bluer than the value of +0.94 given in
Allen (1999) for such a spectral type, suggesting that the companion is of earlier spectral type.

\section{WDS 05248-5219, I 345, HIP 25298}

The brighter component of the wide pair $\theta$ Pic was found to be double in 1900 by Innes (1901) using the 18-inch McClean refractor at Cape Town. The pair has continued to close since then but was last observed in 1993 about a year before periastron passage. The last 80 degrees of motion have remained unobserved and measurements are urgently needed to confirm the validity of the orbit presented here. Nördstrom \& Andersen (1985) have found that one of the stars in this close pair is a double-lined spectroscopic binary. Although they did not derive an orbit the velocity amplitudes of the two stars indicates a mass ratio of $0.76 \pm 0.02$.

Houk (1978) gives the spectral type as A2V whereas the WDS lists A0V. The parallax is small (6.11 mas) 
Table 4. Ephemerides for five visual binaries.

\begin{tabular}{lllllllllll}
\hline Date & \multicolumn{2}{c}{ HdO181 } & \multicolumn{2}{c}{ Slr1 } & \multicolumn{2}{c}{ I 345 } & \multicolumn{3}{c}{ I 10 } & \multicolumn{2}{c}{ HJ 5014 } \\
\hline 2001 & 56.9 & 0.29 & 240.5 & 0.27 & 306.6 & 0.11 & 341.1 & 1.04 & 6.7 & 1.70 \\
2002 & 54.2 & 0.30 & 225.0 & 0.26 & 298.8 & 0.12 & 338.9 & 1.01 & 6.2 & 1.70 \\
2003 & 51.6 & 0.30 & 208.8 & 0.26 & 292.1 & 0.13 & 336.5 & 0.97 & 5.7 & 1.70 \\
2004 & 49.1 & 0.31 & 193.5 & 0.28 & 286.2 & 0.14 & 333.9 & 0.93 & 5.3 & 1.70 \\
2005 & 46.7 & 0.32 & 180.2 & 0.30 & 281.1 & 0.15 & 331.1 & 0.87 & 4.8 & 1.71 \\
2006 & 44.3 & 0.32 & 169.2 & 0.33 & 276.6 & 0.16 & 327.7 & 0.80 & 4.3 & 1.71 \\
2007 & 42.1 & 0.33 & 160.1 & 0.36 & 272.7 & 0.17 & 323.7 & 0.73 & 3.8 & 1.71 \\
2008 & 39.9 & 0.33 & 152.5 & 0.40 & 269.2 & 0.18 & 318.9 & 0.66 & 3.4 & 1.71 \\
2009 & 37.8 & 0.34 & 146.0 & 0.43 & 266.0 & 0.19 & 312.8 & 0.58 & 2.9 & 1.72 \\
2010 & 35.8 & 0.35 & 140.4 & 0.46 & 263.2 & 0.20 & 305.0 & 0.51 & 2.4 & 1.72 \\
\hline
\end{tabular}

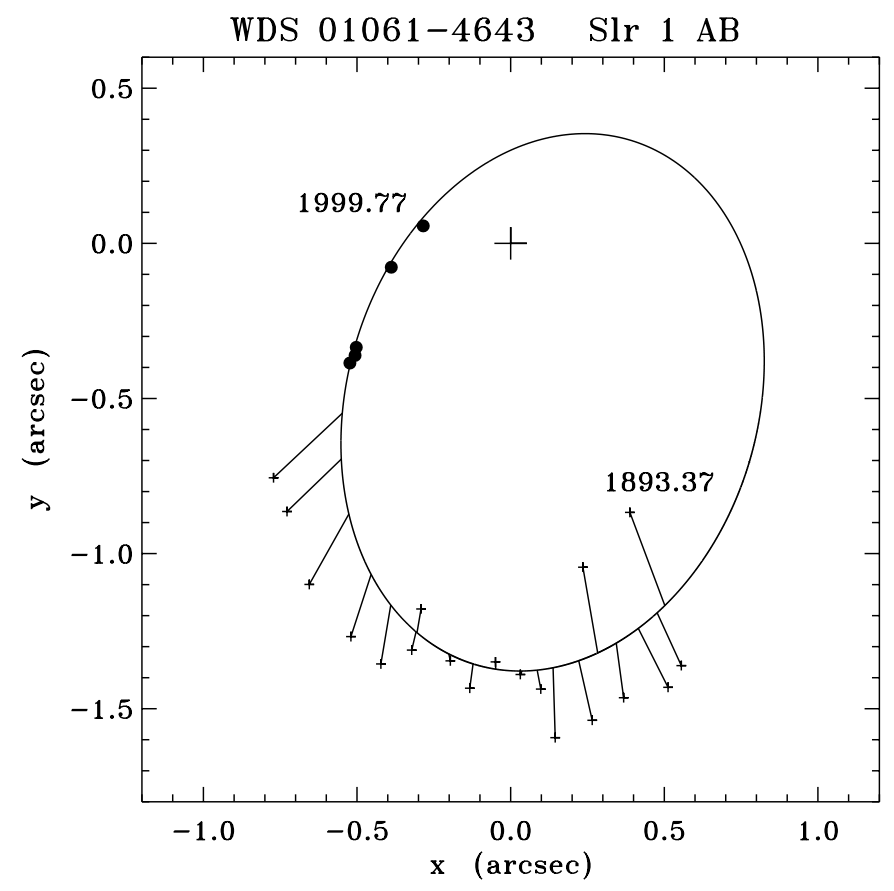

Fig. 2. The apparent orbit of Slr1, period $=195$ years.

but significant $( \pm 0.53$ mas $)$ whilst the dynamical parallax from Baize-Romani is 5.4 mas. The WDS gives the individual $V$ magnitudes as 6.26 and 6.90 .

The physically connected third star (HIP 25303) is 39 arc seconds distant. It has the same parallax and proper motion in declination as I 345 within the Hipparcos errors. The proper motion in right ascension differs by 6 standard errors, possibly reflecting the orbital motion with HIP 25298. It is also given in Simbad as A0V with $B-V=$ +0.07 . Nördstrom \& Andersen suspect that this star has variable radial velocity so $\theta$ Pic is perhaps a quintuple system.

\section{WDS 08447-5443, I 10, HIP 42913}

$\delta$ Velorum (or Argûs as it was then) was first found to be double by Solon Bailey on 1894 April 30 using the 13-inch refractor at Arequipa. Innes (1895) had independently discovered $\delta$ Velorum B, assigning it the designation I 10.

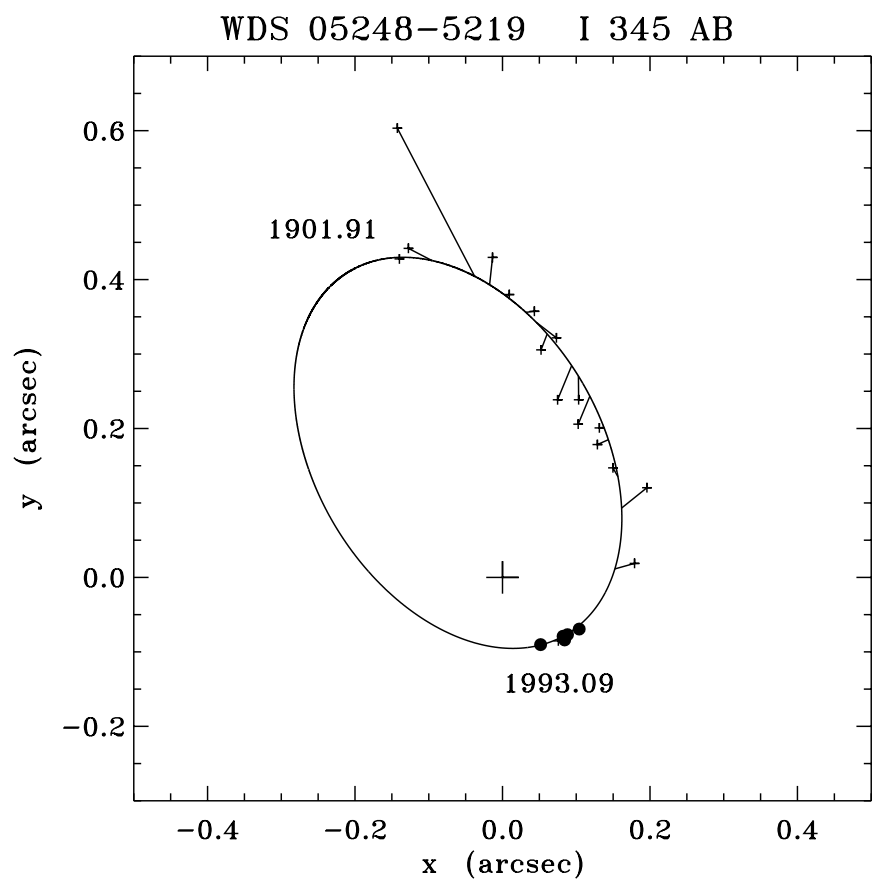

Fig. 3. The apparent orbit of I 345, period $=191$ years.

In the early 1970's Hanbury Brown et al. (1974) were using the Intensity Interferometer at Narrabri for the determination of the diameters of bright stars. A number, including $\delta$ Vel were found to be double at separations of 10 mas and greater. Using the Anglo Australian Telescope and the Imperial College speckle interferometer (Morgan et al. 1982) to try and resolve some of these stars, led to a paper by Tango et al. (1979). The authors stated that the subsequent fringes found in the autocorrellograms of $\delta$ Vel which indicated a companion at a distance of about 0 .' 6 could not be due to that of star $B$ "both because of its distance (the field of the camera was only about $3^{\prime \prime}$ square) and because it is 4 mag fainter than the primary". In fact, we believe that $B$ at this time was at a distance of 0 .' 6 , having closed more quickly than had been expected. 
Table 5. Observational data for five visual binaries.

WDS 00090-5400 = HdO181 (precession negligible)

\begin{tabular}{lllllll}
\hline Epoch & $\theta$ & $\rho$ & $n$ & Obs. & $\Delta \theta$ & $\Delta \rho$ \\
\hline 1897.93 & 300 & 1.2 & 1 & Cly & +3.9 & +0.67 \\
1901.19 & 292.0 & 0.73 & 3 & I & -1.3 & +0.21 \\
1913.79 & 284.7 & 0.39 & 1 & Hu & +3.4 & -0.09 \\
1925.15 & 270.0 & 0.52 & 7 & Daw3, B4 & +1.7 & +0.09 \\
1931.99 & 258.9 & 0.45 & 21 & Vou8, B10, Daw3 & -0.1 & +0.05 \\
1936.48 & 249.9 & 0.47 & 14 & Fin6, Vou4, B4 & -2.3 & +0.09 \\
1942.62 & 245.9 & 0.47 & 4 & Vou & +4.3 & +0.11 \\
1947.44 & 232.2 & 0.44 & 4 & B & +0.1 & +0.10 \\
1955.50 & 214.3 & 0.32 & 10 & B & +0.7 & +0.01 \\
1960.45 & 201.2 & 0.32 & 6 & B & +0.8 & +0.03 \\
1964.57 & 183.7 & 0.30 & 6 & B & -4.6 & +0.02 \\
1975.73 & 153.3 & 0.20 & 3 & Wor & +3.2 & -0.05 \\
1978.52 & 142.0 & 0.23 & 10 & Hei6, Wor4 & +2.7 & -0.02 \\
1983.37 & 116.2 & 0.21 & 3 & Ary1, Hei2 & -3.7 & -0.03 \\
1989.94 & 93.5 & 0.252 & 1 & McA & -0.2 & +0.004 \\
1990.03 & 100.6 & 0.16 & 2 & Hei & +7.3 & -0.09 \\
1991.08 & 88.6 & 0.247 & 2 & Hrt1, Hip & -0.8 & -0.004 \\
1991.71 & 84.1 & 0.256 & 1 & Hrt & -2.9 & +0.003 \\
1999.77 & 62.2 & 0.28 & 2 & Hor & +1.8 & -0.01 \\
\hline
\end{tabular}

WDS 01061-4643 = Slr1; precession: $+0.0023(t-2000)$

\begin{tabular}{lllllll}
\hline Epoch & $\theta$ & $\rho$ & $n$ & Obs. & $\Delta \theta$ & $\Delta \rho$ \\
\hline 1893.37 & 24.1 & 0.95 & 9 & Slr & +0.8 & -0.32 \\
1895.53 & 22.2 & 1.47 & 10 & Tb4, Slr6 & +0.4 & +0.19 \\
1900.64 & 19.7 & 1.52 & 14,10 & Gil1, Slr2, Lun2, & & \\
& & & & Dob4, 0, Tb5 & +1.2 & +0.21 \\
1906.38 & 14.1 & 1.51 & 12 & Dob7, Hst1, Tb4 & -0.8 & +0.18 \\
1911.04 & 12.7 & 1.07 & 2 & I & +0.6 & -0.28 \\
1915.71 & 9.8 & 1.56 & 7 & Vou4, Daw3 & +0.4 & +0.20 \\
1921.90 & 5.2 & 1.60 & 8 & Daw & -0.6 & +0.23 \\
1925.69 & 3.9 & 1.44 & 13 & Vou8, Fin2, I1, & & \\
& & & & Cta2 & +0.3 & +0.06 \\
1929.28 & 1.3 & 1.39 & 18 & I1, Rst2, Vou7, & & \\
& & & & Bru4, Wal4 & -0.2 & +0.01 \\
1935.49 & 357.9 & 1.35 & 13,12 & B5, 4, Vou4, Smw4 & -0.1 & -0.02 \\
1940.86 & 354.7 & 1.44 & 4 & B & -0.1 & +0.08 \\
1946.71 & 351.7 & 1.36 & 27 & Woy7, Gtb6, Hir3, & & \\
& & & & Vou4, B4, Smw3 & +0.3 & +0.02 \\
1954.68 & 346.1 & 1.214 & 1 & The (phot) & -0.2 & -0.08 \\
1954.93 & 346.2 & 1.35 & 4 & Chu & 0.0 & +0.06 \\
1961.78 & 342.7 & 1.42 & 3 & Mro1, Pma2 & +1.2 & +0.19 \\
1967.75 & 337.7 & 1.37 & 7 & Knp3, Nbg4 & +0.8 & +0.21 \\
1976.57 & 329.2 & 1.28 & 5 & Wor3, Hln2 & +0.3 & +0.26 \\
1982.66 & 319.9 & 1.13 & 2 & Ary & -1.7 & +0.24 \\
1986.86 & 314.4 & 1.08 & 2 & Sca & -0.6 & +0.31 \\
1990.42 & 306.4 & 0.650 & 2 & McA1, Hrt1 & -1.0 & -0.011 \\
1991.25 & 305.5 & 0.622 & 1 & Hip & +0.2 & -0.011 \\
1991.72 & 303.7 & 0.604 & 2 & Hrt & -0.3 & -0.012 \\
1996.82 & 281.2 & 0.396 & 1 & Hor & -1.3 & -0.020 \\
1999.77 & 258.8 & 0.29 & 3 & Hor & +2.0 & -0.01 \\
\hline & & & & & &
\end{tabular}


Table 5. continued.

WDS 05248-5219 $=$ I 345; precession $=+0.0090(t-2000)$

\begin{tabular}{lllllll}
\hline Epoch & $\theta$ & $\rho$ & $n$ & Obs. & $\Delta \theta$ & $\Delta \rho$ \\
\hline 1901.91 & 198.1 & 0.45 & 5 n & I & -0.1 & 0.00 \\
1912.59 & 196.1 & 0.46 & 2 & I & +3.4 & +0.02 \\
1925.12 & 193.3 & 0.62 & 2 & I & +7.9 & +0.21 \\
1929.43 & 181.8 & 0.43 & 10 & Vou4, Fin6 & -0.8 & +0.04 \\
1933.86 & 178.6 & 0.38 & 9 & Vou5, B4 & -0.9 & 0.00 \\
1939.82 & 173.1 & 0.36 & 4 & Smw & -1.8 & 0.00 \\
1942.74 & 167.2 & 0.33 & 3 & Vou & -5.3 & -0.02 \\
1946.03 & 170.3 & 0.31 & 4 & B & +0.8 & -0.02 \\
1953.49 & 162.6 & 0.25 & 6 & Fin & +0.8 & -0.05 \\
1955.66 & 156.6 & 0.26 & 4,3 & Fin3, 2, B1 & -2.6 & -0.03 \\
1959.51 & 153.5 & 0.23 & 7 & B & -0.5 & -0.04 \\
1964.81 & 146.8 & 0.24 & 6 & Fin2, B4 & +1.0 & 0.00 \\
1966.77 & 144.2 & 0.22 & 3 & Knp2, Nbg1 & +2.0 & -0.01 \\
1972.09 & 134.5 & 0.21 & 5 & Wor & +3.7 & 0.00 \\
1976.113 & 121.6 & 0.23 & 2 & Wor & +1.6 & +0.04 \\
1983.147 & 96.0 & 0.18 & 3 & Wor & +1.7 & +0.03 \\
1987.90 & single & & 4 & Sca & $(69.7$ & $0.13)$ \\
1989.939 & 56.3 & 0.125 & 1 & McA & 0.0 & +0.004 \\
1990.914 & 48.9 & 0.117 & 1 & Hrt & -0.2 & +0.001 \\
1991.17 & single & & 2 & Hei & $(47.1$ & $0.11)$ \\
1991.25 & 45.1 & 0.119 & 1 & Hip & -1.4 & +0.005 \\
1991.727 & 41.6 & 0.114 & 1 & Hrt & -1.0 & +0.002 \\
1993.09 & 29.8 & 0.104 & 1 & Hrt & -0.8 & -0.001 \\
\hline
\end{tabular}

WDS 08447-5443 = I 10; precession $=+^{\circ} .0073(t-2000)$

\begin{tabular}{lllllll}
\hline Epoch & $\theta$ & $\rho$ & $n$ & Obs. & $\Delta \theta$ & $\Delta \rho$ \\
\hline 1895.35 & 161.1 & - & 4 & Tb & -12.0 & - \\
1895.74 & 177.1 & 2.26 & 9 & Bys2, I3, Slr3, See1 & +4.2 & +0.18 \\
1900.22 & 174.6 & 2.31 & 6 & I4, Lun2 & +3.8 & -0.01 \\
1902.12 & 169.6 & - & 1 & Tb & -0.4 & - \\
1908.2 & 170.5 & 2.9 & 1 & Hst & +2.8 & +0.27 \\
1912.96 & 165.3 & 2.72 & 16 & I8, Daw3, Vou5 & -0.9 & -0.05 \\
1917.89 & 164.4 & 3.00 & 14 & I2, Daw8, Vou4 & -0.4 & +0.14 \\
1924.95 & 161.8 & 3.03 & 14 & Joh2, Vou8, Cta3, & & \\
& & & & Bru4 & -1.0 & +0.12 \\
1928.88 & 162.3 & 2.80 & 12 & Vou4, Fin2, Wal6 & +0.6 & -0.10 \\
1935.53 & 159.5 & 2.92 & 5 & B4, Smw1 & -0.4 & +0.09 \\
1945.07 & 157.3 & 2.51 & 11 & Vou4, Woy3, Gtb4 & +0.4 & -0.09 \\
1953.0 & 154.2 & 2.21 & 2 & The & +0.4 & -0.07 \\
1978.92 & 64.7 & 0.627 & 2 & Tng & -50.0 & -0.004 \\
1990.5 & 9.2 & 0.725 & 1 & Hip & -0.2 & +0.020 \\
1991.6 & 4.4 & 0.757 & 1 & Hip & -0.3 & -0.017 \\
1992.5 & 0.6 & 0.774 & 1 & Hip & -0.8 & -0.054 \\
1999.782 & 344.2 & 1.06 & 1 & Hor & +0.6 & +0.00 \\
\hline
\end{tabular}


Table 5. continued.

WDS 18068-4325 = HJ 5014; precession $=-0.0077(t-2000)$

\begin{tabular}{|c|c|c|c|c|c|c|}
\hline Epoch & $\theta$ & $\rho$ & $n$ & Obs. & $\Delta \theta$ & $\Delta \rho$ \\
\hline 1836.67 & 247.8 & 1.0 & 4 & $\mathrm{Hj}$ & +0.3 & +0.12 \\
\hline 1857.28 & 134.0 & 0.55 & 2 & Jc & -0.8 & +0.08 \\
\hline 1880.49 & 80.9 & 0.71 & 2 & R1, Cru1 & +1.4 & -0.44 \\
\hline 1887.46 & 72.6 & 1.35 & 4 & Pol & -0.2 & +0.09 \\
\hline 1892.84 & 69.1 & 1.06 & 11,10 & Slr9, Pic1, 0, Bys1 & +0.7 & -0.27 \\
\hline 1896.55 & 64.6 & 1.56 & 9 & Slr6, Cog2, See1 & -0.9 & +0.20 \\
\hline \multirow[t]{2}{*}{1902.33} & 63.1 & 1.57 & 9 & Lun1, Dob3, I1, & & \\
\hline & & & & Tb1, Sct3 & +1.7 & +0.16 \\
\hline 1908.42 & 58.7 & 1.62 & 4 & Tb3, Ngl1 & +1.4 & +0.17 \\
\hline 1914.73 & 53.1 & 1.58 & 17 & I2, Hu2, Daw9, Vou4 & -0.2 & +0.09 \\
\hline 1920.65 & 50.1 & 1.69 & 3 & Daw & +0.5 & +0.18 \\
\hline 1925.19 & 45.6 & 1.63 & 13 & I3, Vou4, B3, Cta3 & -1.3 & +0.10 \\
\hline \multirow[t]{2}{*}{1929.28} & 43.9 & 1.59 & 29 & Vou8, Bru4, B6, & & \\
\hline & & & & Daw4, Wal7 & -0.6 & +0.05 \\
\hline 1933.62 & 40.8 & 1.52 & 14 & Daw6, Vou4, B4 & -1.2 & -0.03 \\
\hline 1936.74 & 39.3 & 1.43 & 15 & Vou4, Smw8, Fin3 & -0.9 & -0.13 \\
\hline \multirow[t]{2}{*}{1941.89} & 36.8 & 1.64 & 11 & Smw4, Ian2, Woh2, & & \\
\hline & & & & Hir3 & -0.6 & +0.07 \\
\hline \multirow[t]{2}{*}{1946.86} & 33.9 & 1.64 & 25 & Woy5, B4, Gtb6, & & \\
\hline & & & & Hir4, Smw2, Woh4 & -0.7 & +0.05 \\
\hline 1949.20 & 32.2 & 1.52 & 4 & $\mathrm{~B}$ & -1.1 & -0.07 \\
\hline 1951.88 & 31.3 & 1.45 & 4 & Lem(phot) & -0.6 & -0.15 \\
\hline 1955.53 & 30.0 & 1.55 & 4 & Hei & +0.1 & -0.05 \\
\hline \multirow[t]{2}{*}{1957.97} & 27.9 & 1.48 & 2 & Lem1(phot), & & \\
\hline & & & & $\operatorname{Vad} 1$ (phot) & -0.7 & -0.13 \\
\hline 1960.29 & 27.4 & 1.53 & 15 & B4, Mro8, Knp3 & +0.1 & -0.08 \\
\hline 1962.33 & 25.4 & 1.40 & 2 & $\operatorname{Vad}($ phot $)$ & -0.9 & -0.22 \\
\hline 1964.06 & 25.2 & 1.64 & 10 & Mro6, Nbg4 & -0.1 & +0.02 \\
\hline 1969.65 & 22.7 & 1.61 & 7 & Mro3, Wor4 & +0.3 & -0.02 \\
\hline \multirow[t]{2}{*}{1975.46} & 19.0 & 1.52 & 11 & Hln3, Vad2(phot), & & \\
\hline & & & & Wor6 & -0.4 & -0.12 \\
\hline \multirow[t]{2}{*}{1977.90} & 17.3 & 1.73 & 5 & Vad1(phot), Hei3, & & \\
\hline & & & & Wrh1 & -0.9 & +0.09 \\
\hline \multirow[t]{3}{*}{1982.79} & 14.9 & 1.69 & 7 & Wrh2, Ary3, & & \\
\hline & & & & Vad1(phot), & & \\
\hline & & & & Pan1(phot) & -0.8 & +0.04 \\
\hline 1986.89 & 13.6 & 1.64 & 4 & Pan2, Jas2 & 0.0 & -0.02 \\
\hline 1989.92 & 12.6 & 1.62 & 2 & Jas2(phot) & +0.5 & -0.05 \\
\hline 1990.34 & 12.9 & 1.663 & 1 & $\mathrm{McA}$ & +1.0 & -0.008 \\
\hline 1991.25 & 11.7 & 1.674 & 1 & Hip & +0.2 & +0.001 \\
\hline 1991.53 & 11.9 & 1.680 & 1 & Tyc & +0.6 & +0.006 \\
\hline 1991.80 & 11.7 & 1.62 & 3 & Jas2(phot), War1 & +0.5 & -0.05 \\
\hline 1999.79 & 8.4 & 1.72 & 1 & Hor & +1.1 & +0.03 \\
\hline
\end{tabular}




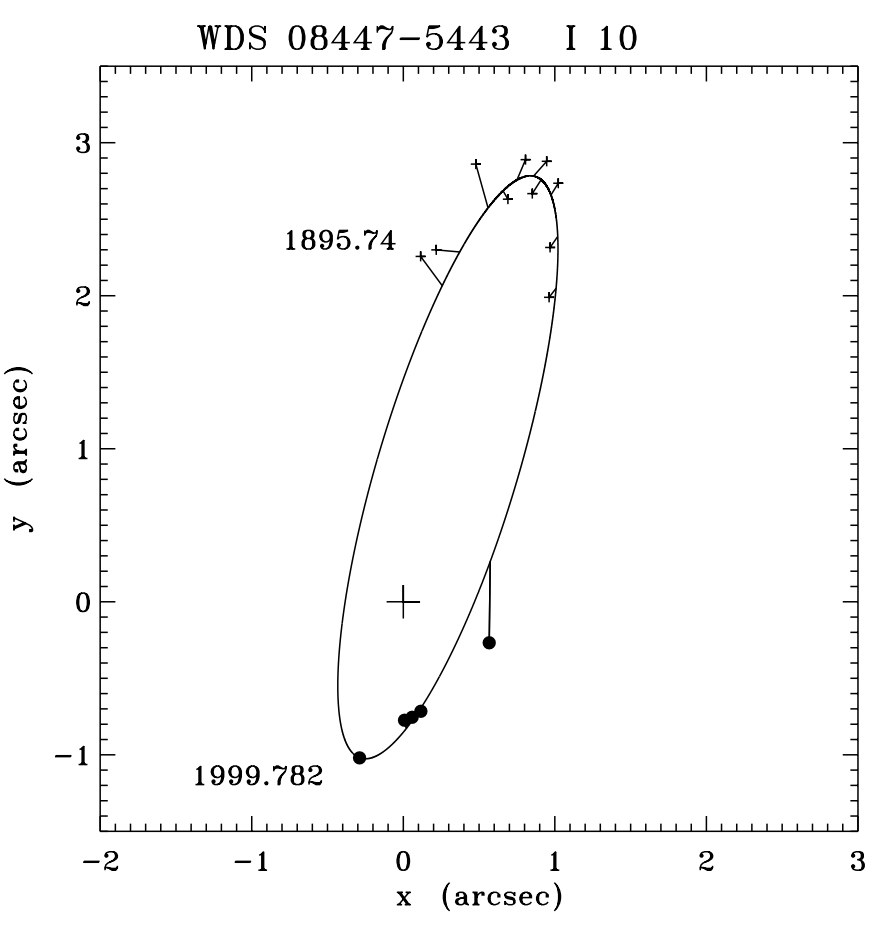

Fig. 4. The apparent orbit of I 10, period $=142$ years.

Professor J. Davis (2000) has indicated that he believes the Narrabri observations do show another component of $\delta$ Vel which is considerably brighter than $B$ which at $V=5.5$ was too faint for the Narrabri instrument at the time. There is no evidence for variability of star $B$ which could explain the observed $\Delta m$ at Narrabri although $B$ has always been a difficult visual object and estimates of the brightness have ranged from 5.0 to 11.0. Barring estimates of 0.7 (perhaps a misidentification) by Tebbutt (1896) and 8.2 (See 1898), the mean visual magnitude difference of $\mathrm{AB}$ from 9 observations is $4.1 \pm 0.4$. Little photometry exists for $B$; it was observed photographically by The (1970) at Lembang in 1952 and 1953 but it was noted as "not separated" and "barely separated" on those occasions, with $\Delta m$ being given as 3.0. Hipparcos recorded a $\Delta H_{\mathrm{p}}$ of $3.58 \pm 0.07$.

In 1996 one of us (RA) realised that the Hipparcos observations of $\delta$ Vel AB showed rapid motion of star $B$ and in correspondence with Dr. W. I. Hartkopf the latter tried several orbital solutions although in each case the measure by Tango et al was difficult to incorporate into the orbit as it gave substantial residuals.

In 1997, the primary star was found to be a visual eclipsing binary with a primary eclipse of $\sim 0.4$ mag every 45.16 days Otero (2000). Otero et al. (2000) conclude that the primary of $\delta \mathrm{Vel}$ is probably a pair of early A stars although there is some evidence that there is a significant $\Delta m$. They speculate that the radial velocity of the eclipsing system could be at least $50 \mathrm{~km} \mathrm{~s}^{-1}$ and consider it possible that the orbit is fairly elliptical. The Hipparcos photometry data for $\mathrm{A}$ in conjunction with the Hipparcos parallax (40.90 \pm 0.38 mas) yields an absolute magnitude of +0.02 . This is $0.6 \mathrm{mag}$ brighter than the expected value

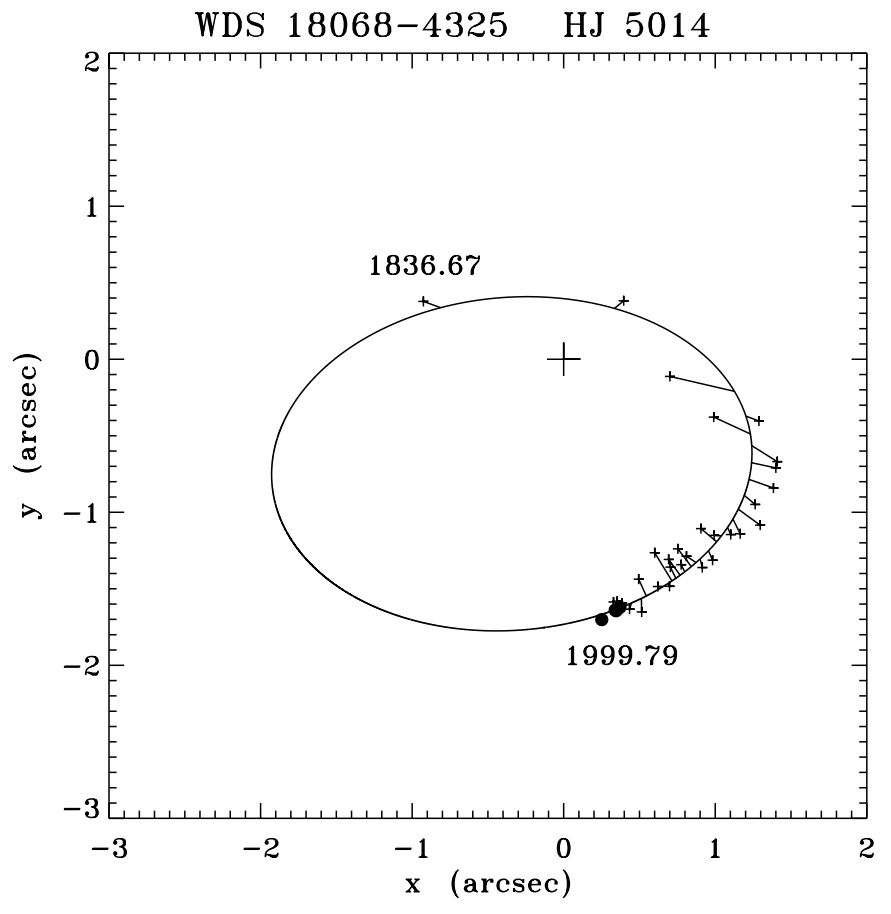

Fig. 5. The apparent orbit of HJ 5014, period $=450$ years.

taken from Allen for an A1V star and confirms the binarity of $\mathrm{A}$.

Only two radial velocity observations of $\delta$ Vel have been found in the literature: a mean value of $+2.1 \mathrm{~km} \mathrm{~s}^{-1}$ (with a range from +13 to $-5 \mathrm{~km} \mathrm{~s}^{-1}$ ) from 5 spectra taken on 3 nights around 1914.0 at Lick Observatory (Lick 1928 ) and $-15.12 \pm 1.19 \mathrm{~km} \mathrm{~s}^{-1}$, a mean of two observations in 1995 Jan. (Grenier et al. 1999). Although Grenier also gives $150 \mathrm{~km} \mathrm{~s}^{-1}$ for $v \sin i$ for both observations, the Bright Star Catalogue quotes a value of $30 \mathrm{~km} \mathrm{~s}^{-1}$ being a mean of two values of 0 and $80 \mathrm{~km} \mathrm{~s}^{-1}$ and notes SB next to the value for radial velocity. Levato (1972) gives a value of $85 \mathrm{~km} \mathrm{~s}^{-1}$. If both components of the eclipsing system (A and a) are A stars then the orbital Doppler motion would vary the apparent broadness of the combined spectral lines but it is difficult to explain the very low $v \sin i$. Even if the bright component was rotationally locked with the orbital period of 45 days the equatorial velocity would be at least $\sim 30 \mathrm{kms}^{-1}$.

We can estimate the size of the apparent orbit of the eclipsing pair using the following relation, adapted from Pourbaix (2001)

$a=3.35729138 \times 10^{-2} K P \sqrt{\left(1-e^{2}\right)} \omega / \sin i$

and assuming that $K=50 \mathrm{kms}^{-1}, e=0.3$ then $a=$ 8 mas. If $K=100$ and $e=0$ then $a=18$ mas which puts the system in range of the Narrabri interferometer (Tango et al. 1979). It is thus possible that the Narrabri observation is that of the eclipsing system since the difference in brightness is also in broad agreement with the comments of Professor Davis.

The orbit derived in Table 1 for the pair Aa-B gives angular residuals of 1 degree or less for the observations 
from 1928 to 1999 and so the Tango observation remains problematic. Even allowing for the fact that the position angle is ambiguous by 180 degrees there is no agreement with the orbital solution presented here. No sign of another visual companion appeared in the observations by Horch et al. (2000) which show only star $B$. This rules out another component wider than 0. . 22 at that epoch and the dynamic range of the detector used was such that a star at least 4 mags fainter could have been seen. The Tango observation suggests that in 1978 at least the star was a very close triple although such systems are rare indeed.

We suggest that the close pair, Aa, consists of two A dwarfs, of spectral types A1V and A5V with masses of 2.7 and $2.0 M_{\odot}$. B has an absolute $V$ magnitude of +3.6 corresponding to an early $\mathrm{G}$ dwarf with mass about $1.0 M_{\odot}$. The systemic mass of $5.7 M_{\odot}$ agrees with that derived from the orbital parameters $a$ and $P$ and the Hipparcos parallax.

Clearly a sustained interferometric, spectroscopic and photometric campaign is necessary to pursue this system further.

\section{WDS 18068-4325 = HJ 5014, HIP 88726}

Discovered by John Herschel, the orbit depends heavily on the first few observations but it is clear than the recent slow direct motion is indicative of a much longer period than that derived by Wierzbinski (1958) whose orbit predicts a separation at discovery of 0 "' 6 . With the 5 -inch telescope which Herschel was using the discovery of such a close pair would be difficult to explain. The current elements, whilst clearly still preliminary, fit all the observed positions well and give a separation of 0 ". 9 for the epoch of discovery (1836).

Houk (1978) gives A5V and A5V adding "the primary and secondary spectra cannot be disentangled".

Whilst moving the spectral types to A7V agrees with the observed Hipparcos $B-V(+0.255 \pm 0.017)$, the $M_{V}$ of about +2.4 for each component and the mass sum both derived from the Hipparcos parallax and the orbital elements $\left(3.6 M_{\odot}\right)$, the error in the mass sum $\left(0.94 M_{\odot}\right)$ means that this is not significant.

\section{Conclusions}

We have calculated orbits for 4 bright southern visual binary systems for the first time. At least two of these systems are of higher multiplicity and further photometry, astrometry and radial velocity measurement is necessary in order to more fully understand these systems.

Acknowledgements. The research made use of the Washington Double Star Catalogue maintained at the USNO. We acknowledge useful discussions with Dr. W. I. Hartkopf and thank
Dr. C. Martin for forming the three individual mean epochs for I 10 from the Hipparcos catalogue.

\section{References}

Alzner, A., \& Argyle, R. W. 2000, IAU Comm., 26 Circ., 142 Argyle, R. W., \& Alzner, A. 2001, IAU Comm., 26 Circ., 143 Alzner, A., \& Argyle, R. W. 2001, IAU Comm., 26 Circ., 144 Cester, B. 1965, Z. Astrophys., 62, 191

Cox, A. N. (ed.) 1999, Allen's Astrophysical Quantities, 4th ed. (Springer, Berlin)

Davis, J. 2000, private communication

Evans, D. S., Menzies, A., Stoy, R. H., \& Wayman, P. A. 1961, Royal Obs. Bull., 48, E393

Fieseler, P. D. 2000, AAVSO, private communication

Grenier, S., Brunage, R., Faraggiano, R., et al. 1999, A\&AS, 135,503

Hanbury Brown, R., Davis, J., \& Allen, L. R. 1974, MNRAS, 167,121

Heintz, W. D. 1978, Double Stars (Reidel, Dordrecht)

Hoffleit, D. 1984, Fourth Catalogue of Bright Stars (New Haven)

Horch, E. P., Dinescu, D. I., Girard, T. M., et al. 1996, AJ, 111, 1681

Horch. E. P., Franz, O. G., \& Ninkov, Z. 2000, AJ, 120, 2638

Houk, N., \& Cowley, A. P. 1975, Michigan Spectral Catalogue, vol. 1 (University of Michigan)

Houk, N. 1978, Michigan Spectral Catalogue, vol. 2 (University of Michigan)

Innes, R. T. A. 1895, MNRAS, 55, 312

Innes, R. T. A. 1901, MNRAS, 61, 575

Lampens, P., Kovalevsky, J., Froeschlé, M., et al. 1997, ESA SP-402, 421

Levato, O. H. 1972, PASP, 84, 584

Publications Lick Observatory 1928, XVI, 135

Mason, B. D., Wycoff, G. L., \& Hartkopf, W. I. (http://ad.usno.navy.mil/ad/wds/wds.html)

Morgan, B. L., Beckman, G. L., Scaddan, R. J., \& Vine, H. A. 1982, MNRAS, 198, 817

Nördstrom, B., \& Andersen, J. 1985, A\&AS, 61, 53

Pickering, W. 1897, Harvard Obs. Circ., 18

Pickering, W. 1902, Harvard Obs. Ann., LX (VII), 195

Pourbaix, D. 2001, A\&A, 369, L22

Otero, S. A. (http://www.kusastro.kyoto-u.ac.jp/vsnet/ Mail/vsnet-newvar/msg00599.html)

Otero, S. A, Fieseler, P. D., \& Lloyd, C. 2001, IBVS, 4999

See, T. J. J. 1898, Astron. Nachr., 146, 225

Sellors, R. P. 1893, MNRAS, 54, 123

Tango, W. J., Davis, R. J., Thompson, R. J., \& Hanbury Brown, R. 1979, Proc. Australian Astro. Soc., 3, 323

Tebbutt, J. 1896, MNRAS, 56, 353

The, P. S. 1970, A\&AS, 1, 357

The Hipparcos and Tycho catalogues 1997 (ESA SP-1200) (ESA, Noordwijk), 1, 59

van Herk, G. 1965, Bull. Astr. Inst. Netherlands, 18, 71

Wierzbinski, S. 1958, J. Obs., 41, 78 